\title{
Long non-coding RNA DILC is involved in sepsis by modulating the signaling pathway of the interleukin-6/signal transducer and activator of transcription 3/Toll-like receptor 4 axis
}

\author{
WEIPING HUANG, LINQIANG HUANG, MIAOYUN WEN, MING FANG, YIYU DENG and HONGKE ZENG \\ Department of Emergency and Critical Care Medicine, Guangdong General Hospital, \\ Guangdong Academy of Medical Sciences, Guangzhou, Guangdong 510080, P.R. China
}

Received March 24, 2017; Accepted November 28, 2017

DOI: $10.3892 / \mathrm{mmr} .2018 .9559$

\begin{abstract}
Sepsis is characterized by systemic inflammatory responses. In the present study, the role of deleted in liver cancer 1 (DILC), interleukin (IL)-6, signal transducer and activator of transcription 3 (STAT3), and Toll-like receptor 4 (TLR4) in the pathogenesis of sepsis was investigated. Reverse transcription-quantitative polymerase chain reaction analysis and western blotting were performed to evaluate the effects of lipopolysaccharide (LPS) on the expression of DILC, IL-6, STAT3, and TLR4, in addition to the effects of DILC and IL-6 on the synthesis of tumor necrosis factor (TNF- $\alpha$ ), chemokine ligand 5 (CCL5), E-selectin and C-X-C motif chemokine receptor 1 (CXCR1). In addition, the regulatory association between DILC, IL-6, STAT3 and TLR4 was investigated. LPS reduced the expression level of DILC, and enhanced the expression of IL-6, STAT3 and TLR4. DILC directly and negatively regulated the synthesis of IL- 6 , as demonstrated by the markedly decreased luciferase activity in cells transfected with a wild-type DILC plasmid. On the other hand, compared with the scramble control, DILC and IL-6 small interfering (si)RNAs significantly suppressed the expression of IL-6, STAT3 and TLR4. In addition, DILC siRNA enhanced the expression of IL-6, STAT3 and TLR4, whereas the expression levels of TNF- $\alpha$, CCL5, E-selectin and CXCR1 in LPS-treated THP-1 cells were downregulated following transfection with DILC and IL-6 siRNAs. In patients with sepsis, DILC expression was inhibited, although the expression levels of IL-6, STAT3 and TLR4 were upregulated. In addition, the expression levels of TNF- $\alpha$, CCL5, E-selectin and CXCR1 in patients with sepsis were higher compared with normal subjects. Therefore,
\end{abstract}

Correspondence to: Professor Hongke Zeng, Department of Emergency and Critical Care Medicine, Guangdong General Hospital, Guangdong Academy of Medical Sciences, 106 Zhongshan Road II, Guangzhou, Guangdong 510080, P.R. China

E-mail: dilcsepsis@126.com

Key words: deleted in liver cancer 1, interleukin-6, signal transducer and activator of transcription 3, Toll-like receptor4, sepsis
DILC may mediate the crosstalk between the cascades of IL-6/STAT3 and TNF- $\alpha$ signaling, indicating that DILC may act as a prognostic biomarker of sepsis, and may serve as a potential therapeutic target for the treatment of sepsis.

\section{Introduction}

Sepsis is one of the most frequent causes of mortality among hospitalized patients. The mortality rate of sepsis is similar to that of myocardial infarction (1). In North America, the number of hospitalized patients (per 100,000 patients) suffering from septicemia or sepsis increased by $70 \%$ between 2001 (2) and 2008 (3), and the incidence of severe postoperative sepsis has increased by three times (from 0.3 to $0.9 \%)(2,3)$. In addition, the incidence of sepsis is particularly high among older people and tends to increase with advancing age (1). In North America, $290 \%$ of the population are unfamiliar with the medical term 'sepsis', whereas the majority of those who understand this term do not know that sepsis is the biggest contributor to mortality (4).

Among the Toll-like receptors (TLRs), TLR4 serves an important role in a variety of inflammatory disorders, including asthma, sepsis and chronic obstructive pulmonary disease. Therefore, the TLR4 signaling pathway may be very important in the regulation of these disorders. It was reported that TAK-242, a new small-molecule cyclohexene derivative, may selectively suppress TLR4 signaling and may lead to beneficial outcomes in a mouse model of endotoxins (5). TLR4 acts as a receptor for a variety of endogenous ligands, including heat shock proteins, hyaluronic acid, fibrinogen and high mobility group box 1 , and the interaction between endogenous ligands and TLR4 is involved in a number of human diseases, including sepsis.

Interleukin (IL)-6 is a multifunctional cytokine released by a variety of cells; however, the level of IL-6 in healthy populations is undetectable (6). In trauma patients, an early increase in IL- 6 may be associated with the magnitude of trauma, the severity of proinflammatory reactions and the incidence of complications (sepsis, multiple organ failure and mortality) $(7,8)$. Similarly, IL-10 is a major anti-inflammatory factor and its expression levels are undetectable within healthy individuals. The expression level of IL-10 is increased in 
patients with sepsis, and correlates with the magnitude of sepsis and the likelihood of mortality (9). The ratio of IL-6/IL-10 has long been used to evaluate the immune system in intensive care unit patients, as such a ratio may reveal the balance between proinflammatory and anti-inflammatory responses (systemic inflammatory syndrome and compensatory anti-inflammatory response) (10). A reduction in the IL-6/IL-10 ratio may affect patient prognosis and the occurrence of postoperative sepsis (11).

As transcripts of $>200$ bp in length, long noncoding (lnc) RNAs do not demonstrate any significant protein-coding capability (12). At present, an increasing amount of data has demonstrated that IncRNAs participate in a variety of pathological and physiological processes, and hence may impact various cellular functions (13). It has been recognized that nuclear factor $(\mathrm{NF})-\kappa \mathrm{B}$ serves as a bridge between cancer and inflammation (14). A recent study demonstrated the critical role of $\mathrm{NF}-\kappa \mathrm{B}$ in the maintenance and spread of cancer stem cells (CSCs) (15). In addition, it has been reported that lnc-deleted in liver cancer 1 (DILC) may induce the interaction between the autocrine IL-6/signal transducer and activator of transcription 3 (STAT3) cascade and tumor necrosis factor (TNF)- $\alpha / N F-\kappa B$ signaling in liver CSCs (LCSCs), thus linking liver inflammation to the dissemination of LCSCs (16). It has been demonstrated that the expression of IL-6 may be inhibited by DILC, whereas the expression of STAT3, a downstream component of the IL-6 signaling pathway, may be enhanced by IL-6 stimulation (16). Furthermore, IL- 6 signaling has been demonstrated to modulate TLR4-dependent inflammatory responses via STAT3 (17). In the present study, the role of DILC, IL-6, STAT3 and TLR4 in the pathogenesis of sepsis was investigated.

\section{Materials and methods}

Sample collection. A total of 36 patients, including 18 patients with sepsis and 18 healthy participants, were involved in the present study. The mean age of all subjects was 56 years old (42-78 years), and the ratio of female to male subjects was 1.0:1.5. Peripheral blood samples from 18 participants diagnosed with sepsis and 18 people free of any health problems, were collected using Lymphocyte Separation Medium (Human) (Applygen Technologies Inc., Beijing, China), and stored at $-80^{\circ} \mathrm{C}$. Written informed consent was obtained from all patients. All data processing and sample collection were approved by the Ethics Committee of Guangdong Academy of Medical Sciences. The present study was performed according to the Declaration of Helsinki.

Peripheral blood mononuclear cell (PMBC) isolation. PBMCs were maintained at $-80^{\circ} \mathrm{C}$ until use. A 70 -mm strainer (Falcon; Corning, Inc., Corning, NY, USA) was utilized for cell passaging to yield single cell suspensions, according to the manufacturer's protocol. Dulbecco's modified Eagle's medium (DMEM; Gibco; Thermo Fisher Scientific, Inc., Waltham, MA, USA) containing $10 \%$ fetal bovine serum (FBS; Thermo Fisher Scientific, Inc.), $100 \mathrm{mg} / \mathrm{ml}$ streptomycin sulfate and $100 \mathrm{U} / \mathrm{ml}$ penicillin sodium was utilized to culture the cells into 48 -well plates with $5 \% \mathrm{CO}_{2}$ at $37^{\circ} \mathrm{C}$. Growth medium was changed every 2 days until the cells were passaged, then transferred to a new dish; at $80 \%$ confluence, the PMBCs were passaged again, and cells at passage 2-3 were utilized for analysis.

Cell culture and transfection. DMEM (Gibco; Thermo Fisher Scientific, Inc.) containing 10\% FBS (Thermo Fisher Scientific, Inc.), $100 \mathrm{mg} / \mathrm{ml}$ streptomycin sulfate and $100 \mathrm{U} / \mathrm{ml}$ penicillin sodium was utilized to maintain THP-1 cells under a humidified atmosphere with $5 \% \mathrm{CO}_{2}$ at $37^{\circ} \mathrm{C}$. DILC mimic (5'-AAT ATCGTCAATGGCTATGGCCGTGAAGCGGGCATGCCC CTGGCCACTAGCCGCCGCATCGCCAAAATCGCCTTT ACCGGCTCCACCTCCACCGGCCGCGTGATTGCCCAG GCCGCAGCCAACAATTTGATCCCCGCCACGCTGGAA CTCGGCGGCAAATCGCCCAACATTTTCTTCGCCGAC GTGATGGACAAAGATGATGGCTTCCTG-3') and its negative control were synthesized by Guangzhou RiboBio Co., Ltd. (Guangzhou, China). When the cells reached $80 \%$ confluence, Lipofectamine $^{\circledR} 2000$ (Thermo Fisher Scientific, Inc.) was used to transfect the DILC mimic or NC into THP-1 cells according to the manufacturer's protocol. The time interval between the completion of transfection and the subsequent experiments was $4 \mathrm{~h}$. The culture cells were also treated with LPS $(1 \mathrm{mg} / \mathrm{ml})$ for $30 \mathrm{~min}$. A total of three independent experiments were carried out.

$R N A$ isolation and reverse transcription-quantitative polymerase chain reaction ( $R T-q P C R)$ analysis. TRIzol reagent (Invitrogen; Thermo Fisher Scientific, Inc.) was used to extract total RNA from THP-1 cells and PBMCs, according to the manufacturer's protocol. A TaqMan micro (mi)RNA reverse transcription kit (Applied Biosystems; Thermo Fisher Scientific, Inc.) was used to reverse transcribe the RNA to DNA (cDNA) with designed primers including primers for DILC (forward: 5'-ATGGCA AAAGATGCCGGTCTAATTG-3'; reverse: 5'-TCGTCC TCGTCATTCTCG-3'), IL-6 (forward: 5'-ATGAACTCC TTCTCCACAAGC-3'; reverse: 5'-CTACATTTGCCGAAG AGCCCTCAGGCTGGACTG-3'), STAT3 (forward: 5'-ACT CCATCGCTGACAAAA-3'; reverse: 5'-CAGTGACCAGGC AGAAGA-3'), and TLR4 (forward: 5'-AGCCACCTCTCT ACCTTAATATTGA-3'; reverse: 5'-CCGAGTGTTAGAATA GGTTAGAAAG-3') synthesized by Applied Biosystems (Thermo Fisher Scientific, Inc.), according to the manufacturer's protocol. The Applied Biosystems 7500 Real-Time PCR system (Applied Biosystems; Thermo Fisher Scientific, Inc.) was used to perform RT-qPCR, with a 20- $\mu 1$ mixture containing $10 \mu \mathrm{l}$ SYBR advantage qPCR Premix (Clontech Laboratories, Inc., Mountainview, CA, USA), $1 \mu 1$ cDNA, $7 \mu 1$ $\mathrm{H}_{2} \mathrm{O}$ and $1 \mu \mathrm{l}$ each forward and reverse primer. The temperature conditions were: $95^{\circ} \mathrm{C}$ for $3 \mathrm{~min}$, followed by 30 cycles of $94^{\circ} \mathrm{C}$ for $40 \mathrm{sec}, 56^{\circ} \mathrm{C}$ for $35 \mathrm{sec}$ and final extension at $72^{\circ} \mathrm{C}$ for $60 \mathrm{sec}$. The relative expression levels of DILC, IL-6, STAT3 and TLR4 were quantified using the standard curve method. U6 (primer sequence forward: 5'-ATGCACTATCATATGCTT ACCGTA-3'; reverse: 5'-AGGCGATTAAGTTGGGTA-3') and NADPH (primer sequence forward: 5'-CCGAGAAGTTTC AGCACATCC-3'; reverse: 5'-TGGCAGTGATAGCGAAG GCT-3') were used internal control for mRNAs, respectively. Relative expression levels of DILC, IL-6, STAT3 and TLR4 were presented using the $2^{-\Delta \Delta \mathrm{Cq}}$ method (18). All reactions were run in triplicate. 
Luciferase assay (promoter). A luciferase assay was performed to examine whether DILC alters the transcriptional ability of the promoter of IL-6. qPCR was performed according to the protocol described above to amplify the full promoter segment from DNA templates collected from PBMCs. IL-6 promoter-luciferase reporter plasmids containing either a wild type (WT) or mutant (MUT) DILC binding site were generated accordingly. Sequencing was performed to confirm the reporters with WT or MUT DILC binding sites. Endonuclease (New England BioLabs, Inc., Ipswich, MA, USA) was used to digest the pcDNA3-control vector and amplified fragments. Subsequently the qPCR products were inserted into the pcDNA3-control vector (Ambion; Thermo Fisher Scientific, Inc.). THP-1 cells were seeded into 96-well plates at a final concentration of $3 \times 10^{4}$ cells $/ \mathrm{ml}$, and transfected with $50 \mathrm{ng}$ pcDNA3-DILC (WT or MUT) via electrophoresis (Lonza Group. Ltd., Basel, Switzerland). A dual-luciferase reporter assay system (Promega Corporation, Madison, WI, USA) was used to determine the firefly and Renilla luciferase activity $48 \mathrm{~h}$ post-transfection. All tests were repeated in triplicate.

Western blot analysis. Ice-cold PBS was used to wash the THP-1 cells twice, and radioimmunoprecipitation buffer (Pierce; Thermo Fisher Scientific, Inc.) was used to lyse cells and tissue sample to isolate protein for $30 \mathrm{~min}$ on ice, according to the manufacturer's protocols. The lysates were subjected to centrifugation for $20 \mathrm{~min}$ at $12,000 \mathrm{x} \mathrm{g}$ at $4^{\circ} \mathrm{C}$. A bicinchoninic acid protein assay kit (Boster Biological Technology, Pleasanton, CA, USA) was used to quantify proteins. SDS-PAGE on a $10 \%$ gel was used to separate $30-\mu$ g protein samples, and electroblotting was employed to transfer the target protein onto a polyvinylidene difluoride (PerkinElmer, Inc., Waltham, MA, USA) membrane for $90 \mathrm{~min}$. To block the membrane, $5 \%$ non-fat milk was applied to the membrane to avoid nonspecific binding for $2 \mathrm{~h}$ at room temperature. The primary antibody anti-IL-6 (1:5,000; cat. no. sc-130326; Santa Cruz Biotechnology Inc., Dallas, TX, USA), primary antibody anti-STAT3 (1:5,000; cat. no. sc-8019; Santa Cruz Biotechnology, Inc.), primary antibody anti-TLR4 (1:5,000; cat. no. sc-52962, Santa Cruz Biotechnology Inc.) and anti- $\beta$-actin (1:10,000; cat. no. sc-47778, Santa Cruz Biotechnology Inc.) were used to incubate the membrane at $4^{\circ} \mathrm{C}$ for $12 \mathrm{~h}$. Horseradish peroxidase (HRP)-labeled anti-mouse goat secondary antibody was used to treat the membrane at $37^{\circ} \mathrm{C}$ for $1 \mathrm{~h}$ (1:3,000; cat. no. 7074; Cell Signaling Technology, Inc.). A chemiluminescence detection system (Pierce; Thermo Fisher Scientific, Inc.) was used to determine the protein bands, and the Bio-Rad Chemi-DocXRS (Bio-Rad Laboratories, Inc., Hercules, CA, US) was utilized to visualize the bands of the target proteins. Each test was performed in triplicate.

ELISA analysis. A total of $50 \mathrm{mM}$ carbonate-bicarbonate buffer supplemented with mouse polyclonal immunoglobulin $\mathrm{G}$ (IgG) anti-TNF- $\alpha$ (cat. no. 6945; Cell Signaling Technology, Inc.)/CCL5 (cat. no. 2988; Cell Signaling Technology, Inc.) /E-selectin (cat. no. 14-0627-82; Thermo Fisher Scientific, Inc., Waltham, MA, USA)/CXCR1 antibodies (1:1,500; cat. no. MA1-201; Thermo Fisher Scientific, Inc.) were used to treat THP-1 cells on 96-well plates at a final concentration of $3 \times 10^{4}$ cells $/ \mathrm{ml}$, and maintained at $4^{\circ} \mathrm{C}$ for $12 \mathrm{~h}$.PBS supplemented with $4 \%$ bovine serum albumin (BSA, LI-COR Biosciences, Lincoln, NE, USA) was used to treat for another $60 \mathrm{~min}$ and PBS with $0.1 \%$ Tween-20 was used to wash the plates. A total of $50 \mu \mathrm{l}$ purified TNF- $\alpha /$ CCL5/E-selectin/CXCR1 antigens and clinical samples were distributed into 96 wells, incubated for $120 \mathrm{~min}$ at $37^{\circ} \mathrm{C}$, and washed and cultured for $60 \mathrm{~min}$ at $37^{\circ} \mathrm{C}$ in $100 \mu \mathrm{PBS}$, containing $1 \% \mathrm{BSA}$ and rabbit monoclonal IgG anti-TNF- $\alpha$ (cat. no. 6945; Cell Signaling Technology, Inc.) /CCL5 (cat. no. 2988; Cell Signaling Technology, Inc.) /E-selectin (cat. no. 14-0627-82; Thermo Fisher Scientific, Inc.) /CXCR1 (cat.no.MA1-201; Thermo Fisher Scientific, Inc.) at a dilution of 1:1,500. HRP-conjugated secondary antibodies (1:3,000; cat. no. 7074; Cell Signaling Technology, Inc.) and 1\% BSA (LI-COR Biosciences) were used to maintain the plate. A microplate reader was used to determine the HRP activity with the use of O-phenylenediamine based on the absorbance at $492 \mathrm{~nm}$, and $\mathrm{H}_{2} \mathrm{O}_{2}$ served as the enzyme substrates. All tests were performed in triplicate.

Statistical analysis. SPSS 13.0 software (SPSS, Inc. Chicago, IL, USA) was used to perform all statistical analyses. All results are presented as the mean \pm standard deviation. Unpaired t-tests and one-way analysis of variance followed by Mann-Whitney $U$ test were used to analyze the significance comparisons among more than two groups. $\mathrm{P}<0.05$ was considered to indicate a statistically significant difference.

\section{Results}

Lipopolysaccharide (LPS) alters the production of inflammatory mediators. LPS, a widely recognized stimulator of inflammation, was used in the present study to treat THP-1 cells. RT-qPCR was performed to determine whether LPS affected the expression of relevant inflammatory cytokines. In particular, the expression levels of DILC, IL-6, STAT3 and TLR4 in the cells treated with or without LPS was measured. As presented in Fig. 1A, the expression of DILC reached a stable state at $4 \mathrm{~h}$ following treatment with LPS; however, the levels of DILC expression were decreased in LPS-treated THP-1 cells compared with control cells. Conversely, the expression levels of IL-6 (Fig. 1B), STAT3 (Fig. 1C) and TLR4 (Fig. 1D) were increased in LPS-treated cells. Consistent with the observation that treatment with LPS upregulated the protein expression levels of STAT3 (19), these results suggested that LPS inhibited the expression of DILC and enhanced the expression of IL-6, STAT3 and TLR4.

Lnc-DILC binds to the promoter of IL-6 and suppresses the transcription of $I L-6$. As presented in Fig. 2A, a putative complementary binding site of lnc-DILC was located within the promoter region of IL-6. To further verify the regulatory association between 1 nc-DILC and the IL- 6 promoter, an oligo mimic (oligoribonucleotide) and its mutated control (oligo mut), in addition to an oligodeoxynucleotide (ODN) decoy and its mutated control (ODN mut), were synthesized based on the sequence of the binding locus (Fig. 2B). Subsequently, the luciferase activity of IL-6 was detected. As presented in Fig. 2C, the luciferase activity in cells transfected with the WT DILC vectors was markedly decreased compared with the control cells, whereas the IL-6 luciferase activity in cells transfected 
A

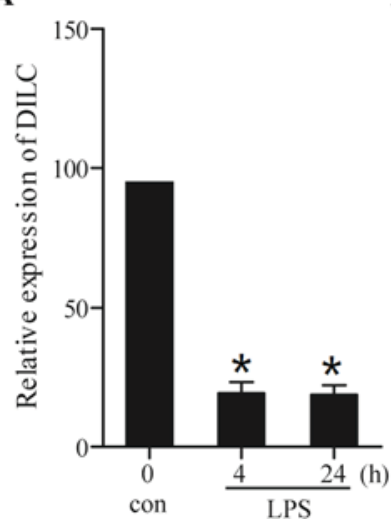

B

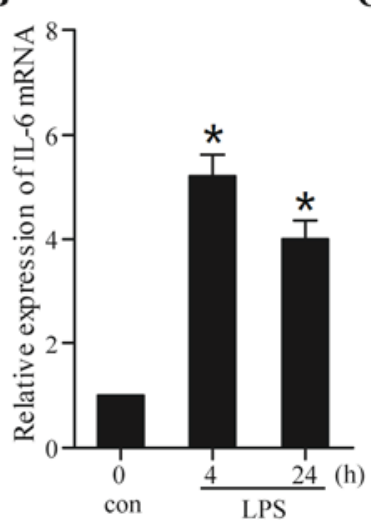

C

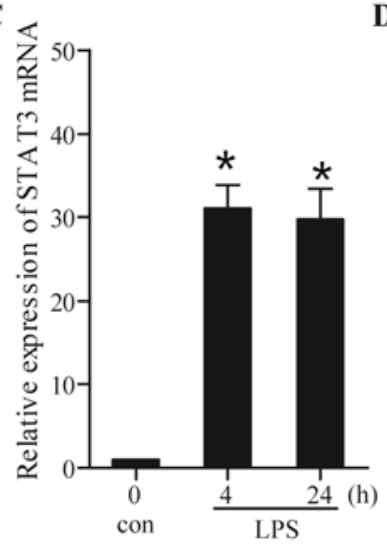

D

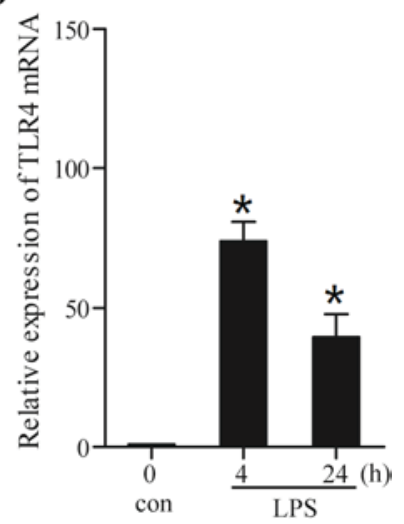

Figure 1. LPS influences the production of inflammatory mediators. (A) DILC expression was significantly lower in cells treated with LPS compared with the control; stable expression of DILC at $4 \mathrm{~h}$ following treatment with LPS was observed. (B) LPS upregulated IL-6 expression levels, which were stable $4 \mathrm{~h}$ following treatment with LPS. (C) Treatment with LPS increased STAT3 production; expression levels of STAT3 were stable 4 h following treatment with LPS. (D) TLR4 was much higher in cells treated with LPS compared with the control, and was stable at $4 \mathrm{~h}$ following treatment with LPS. * $<0.05$ vs. con. DILC, deleted in liver cancer 1; IL, interleukin; LPS, lipopolysaccharide; STAT3, signal transducer and activator of transcription 3; TLR4, Toll-like receptor 4; con, control.

with the mutant DILC vectors remained unchanged, indicating that lnc-DILC bound to the promoter of IL-6 and suppressed its production.

Lnc-DILC alters the expression of IL-6, STAT3 and TLR4 in LPS-treated THP-1 cells. To further understand whether DILC affects the productions of inflammatory cytokines involved in sepsis, LPS-treated THP-1 cells were transfected with WT or MUT DILC vectors, and the mRNA expression level of IL-6, STAT3 and TLR4 in these cells was measured using RT-qPCR and western blot analyses. Transfection with WT DILC significantly reduced the mRNA levels of IL-6 and TLR4, whereas transfection with mutant DILC did not alter the mRNA expression levels of IL-6 and TLR4 (Fig. 3). In addition, it was observed that the WT and MUT DILC vectors demonstrated a minimal effect on the mRNA expression levels of STAT3 (Fig. 3). Furthermore, the protein expression levels of IL-6, STAT3, phosphorylated (p)-STAT3 and TLR4 in these cells was measured and it was observed that transfection with WT DILC reduced the protein expression levels of IL-6, STAT3, p-STAT3 and TLR4, whereas transfection with MUT DILC did not alter the protein expression levels of these genes (Fig. 3).

Inc-DILC alters the levels of STAT3 and TLR4 by regulating the expression of $I L-6$. To investigate the effects of DILC on the expression of IL-6, STAT3 and TLR4, the mRNA and protein expression levels of IL-6, STAT3 and TLR4 in THP-1 cells transfected with DILC and IL-6 siRNAs were measured. As presented in Fig. 4, transfection with DILC siRNA significantly upregulated the mRNA levels of IL-6 and TLR4, whereas transfection with IL-6 siRNA significantly reduced the mRNA level of IL-6 and TLR4. Conversely, transfection with DILC and IL-6 siRNAs did not alter the mRNA expression of STAT3 (Fig. 4B). In addition, transfection with DILC siRNA upregulated the protein expression levels of IL-6, STAT3, p-STAT3 and TLR4; however, transfection with IL-6 siRNA downregulated the protein expression levels of IL-6, STAT3, p-STAT3 and TLR4 (Fig. 4D).
Inc-DILC alters the levels of TNF- $\alpha, C C L 5$, E-selectin and CXCRI by regulating the expression of $I L-6$. To investigate the effects of lnc-DILC on the expression of TNF- $\alpha$, CCL5, E-selectin and CXCR1, THP-1 cells were stimulated with LPS and transfected with the WT or MUT DILC vectors in conjunction with DILC and IL-6 siRNAs. Subsequently, the expression levels of TNF- $\alpha$, CCL5, E-selectin and CXCR1 within these cells was determined using RT-qPCR and western blot analyses. As presented in Fig. 5, the levels of TNF- $\alpha$ (Fig. 5A), CCL5 (Fig. 5B), E-selectin (Fig. 5C) and CXCR1 (Fig. 5D) in the cells transfected with the WT DILC and IL- 6 siRNA were significantly reduced, whereas the levels of these cytokines were significantly increased following transfection DILC SiRNA.

Expression of sepsis-associated genes varies between septic and healthy groups. In the present study, 36 subjects, including 18 patients with sepsis and 18 healthy control subjects, were recruited and the expression levels of DILC, IL-6, STAT3, TLR4, TNF- $\alpha$, CCL5, E-selectin and CXCR1 in these subjects were measured. As presented in Fig. 6, the sepsis group exhibited decreased levels of DILC, and increased levels of IL-6 and TLR4, compared with the healthy group; however, the levels of STAT3 between the two groups exhibited no significant difference. Additionally, the levels of TNF- $\alpha$ (Fig. 7A), CCL5 (Fig. 7B), E-selectin (Fig. 7C) and CXCR1 (Fig. 7D) were higher in the sepsis group compared with the control group.

\section{Discussion}

The interaction between lnc-DILC and the promoter region of IL-6 was observed in the present study. In addition, the effects of lnc-DILC overexpression and lnc-DILC knockdown, which may affect LCSC expansion, STAT3 activation and IL-6 transcription, were eliminated by the ODN decoy and the oligo mimic of lnc-DILC, respectively, thus suggesting that the interaction between Inc-DILC and the promoter of IL-6 exerted an inhibitory effect on LCSC expansion by suppressing the IL-6/STAT3 axis (15). During the expansion of LCSCs, it 
A

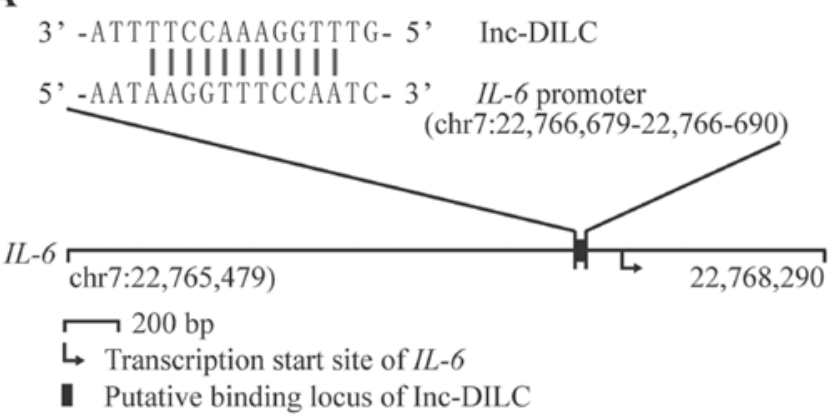

B

\begin{tabular}{|c|c|}
\hline$I L-6$ promoter & 5' -AAGGTTTCCAA- 3 \\
\hline nc-DILC oligo mimics & 3' -UUCCAAAGGUU- 5' \\
\hline Inc-DILC oligo mut & 5' -CACAUCAUGUU- 3 \\
\hline Inc-DILC & 5' - \\
\hline Inc-DIL & \\
\hline Inc-DILC decoy & TAGTAGA \\
\hline
\end{tabular}

C

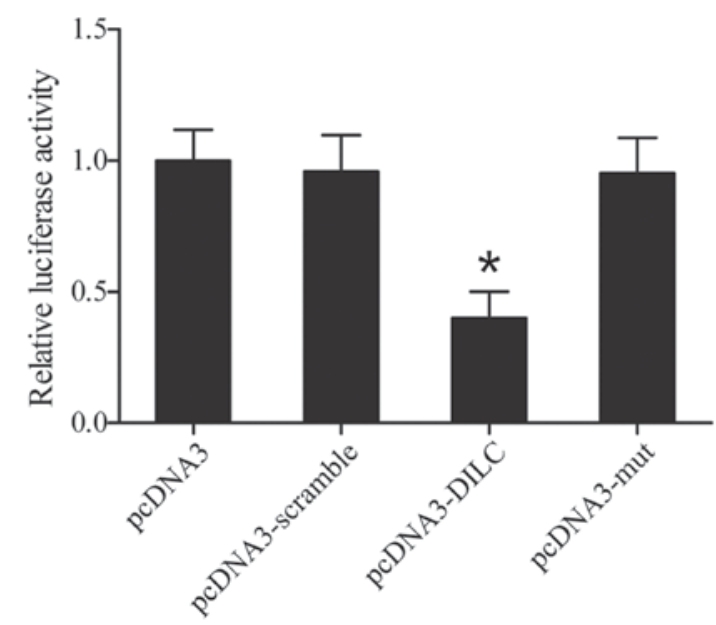

Figure 2. Lnc-DILC binds to the promoter of IL-6 and suppresses the transcription of IL-6. (A) Sequence and location of the postulated lnc-DILC binding locus in the IL-6 promoter. (B) Sequences of RNA oligoribonucleotides and DNA decoy (oligodeoxyonucleotides) used in the present study. (C) Luciferase activity in cells was markedly decreased following transfection with vectors containing wild-type DILC. ${ }^{*} \mathrm{P}<0.05$. DILC, deleted in liver cancer 1; IL, interleukin; lnc, long non-coding; mut, mutant.

has been suggested that lnc-DILC serves as a bridge linking $\mathrm{NF}-\kappa \mathrm{B}$ signaling to the autocrine IL-6/STAT3 cascade (15). It has additionally been demonstrated that lnc-DILC may inhibit the expansion of LCSCs by suppressing the IL-6/STAT3 signaling pathway. Additionally, the elevated expression levels of IL-6 have been revealed to induce the expansion of CSCs by stimulating the expression of STAT3 in hepatocellular carcinoma, and colon, lung, breast and prostate cancers (20-23). In addition, a study demonstrated the ability of STAT3 to enhance the expression of IL-6 in cancer cells (24). For example, the mRNA levels of IL-6 increased in the tumor tissues of gp130 mutant mice under abnormal stimulation with STAT3 (25). Ogura et al (26) reported that the expression levels of IL-6 and other cytokines may be lowered by suppressing the activity of STAT3 in fibroblasts and macrophages (26). In addition, a study suggested that treatment with STAT3-siRNA may
A

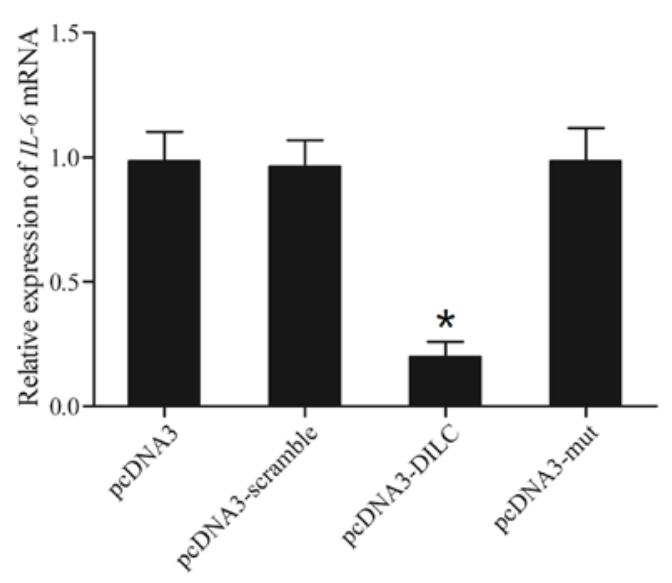

B

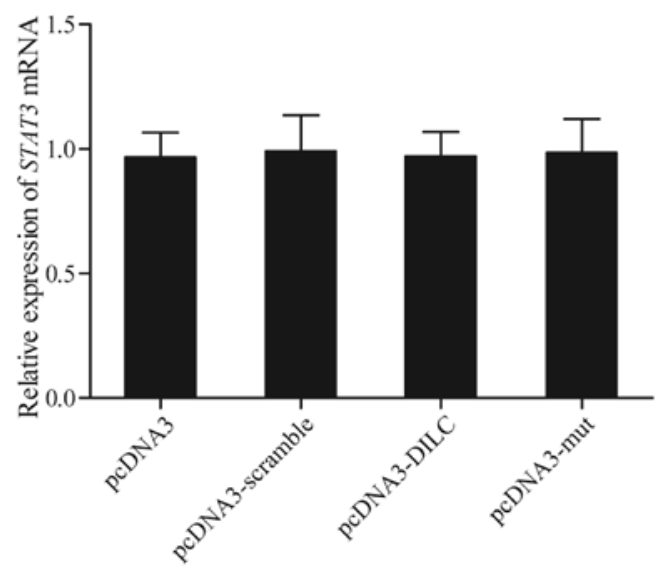

C

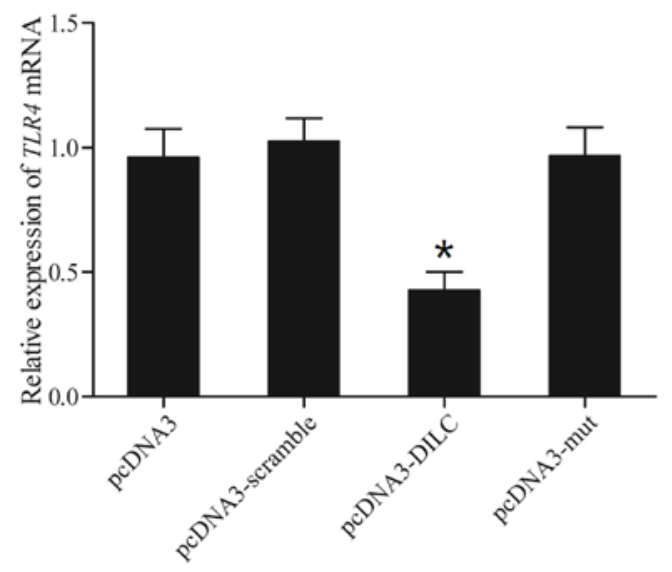

D

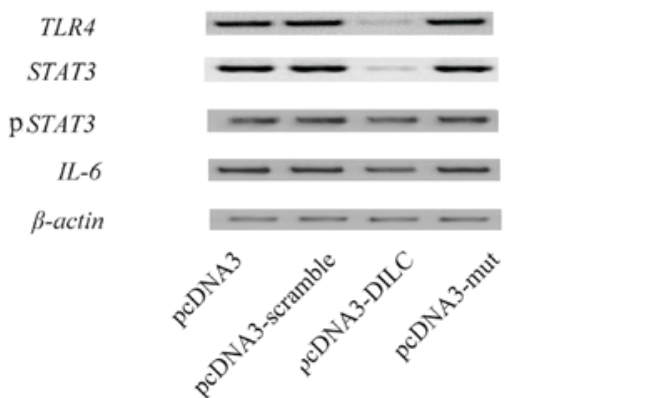

Figure 3. Plasmids containing DILC were transfected into cells treated with LPS. (A) IL-6 mRNA was under-expressed in cells transfected with DILC. (B) STAT3 mRNA expression levels were much lower in cell transfected with DILC. (C) TLR4 mRNA expression levels were decreased in cells following the introduction of DILC. * $\mathrm{P}<0.05$ vs. pcDNA3. (D) IL-6, STAT3/p-STAT3 and TLR4 protein expression levels were decreased in cells transfected with DILC. DILC, deleted in liver cancer 1; IL, interleukin; mut, mutant; p, phosphorylated; STAT3, signal transducer and activator of transcription 3; TLR4, Toll-like receptor 4 . 
A

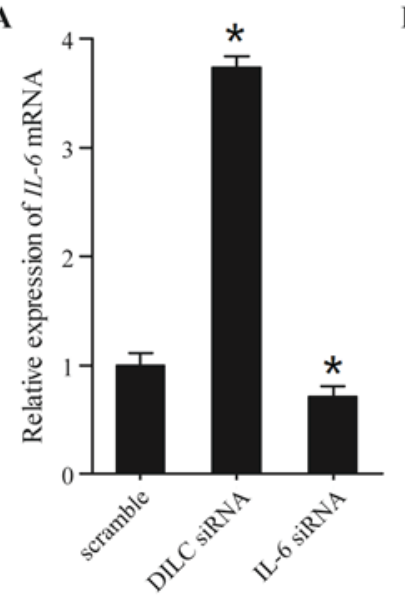

B

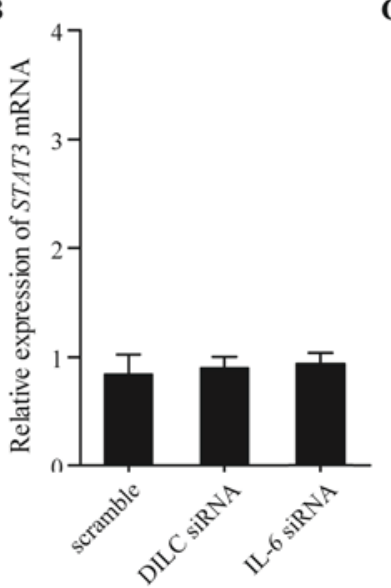

C

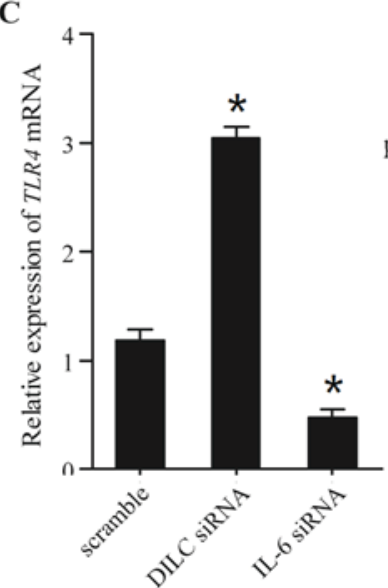

D

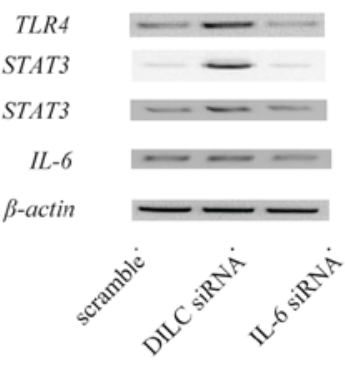

Figure 4. IL-6, STAT3 and TLR4 expression levels in cells transfected with DILC siRNA and IL-6 siRNA. (A) DILC siRNA increased, IL-6 siRNA decreased IL-6 mRNA expression levels. (B) STAT3 mRNA expression levels in the DILC siRNA group were much higher, but much lower in IL-6 siRNA. (C) TLR4 mRNA expression levels were upregulated in the DILC siRNA-treated group, but downregulated in the IL-6 siRNA group. *P<0.05 vs. scramble. (D) IL-6, STAT3/p-STAT3 and TLR4 protein expression levels were increased following transfection with DILC siRNA, and decreased subsequent to transfection with IL-6 siRNA. DILC, deleted in liver cancer 1; IL, interleukin; mut, mutant; p, phosphorylated; STAT3, signal transducer and activator of transcription 3; siRNA, small interfering RNA; TLR4, Toll-like receptor 4.

A

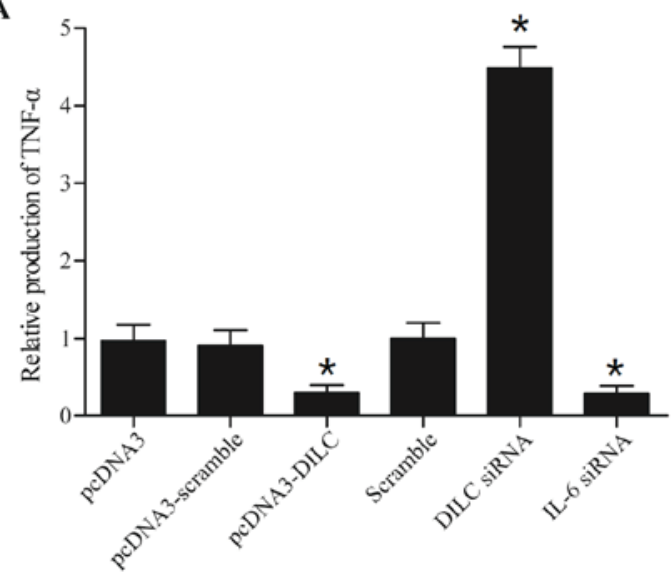

C

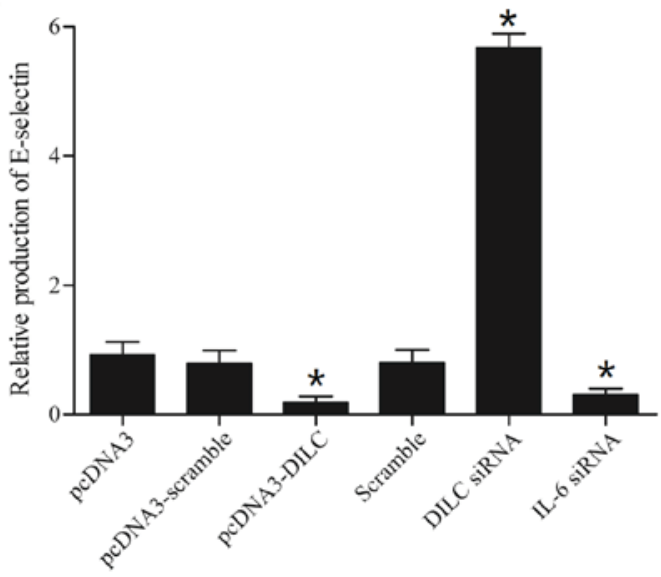

B

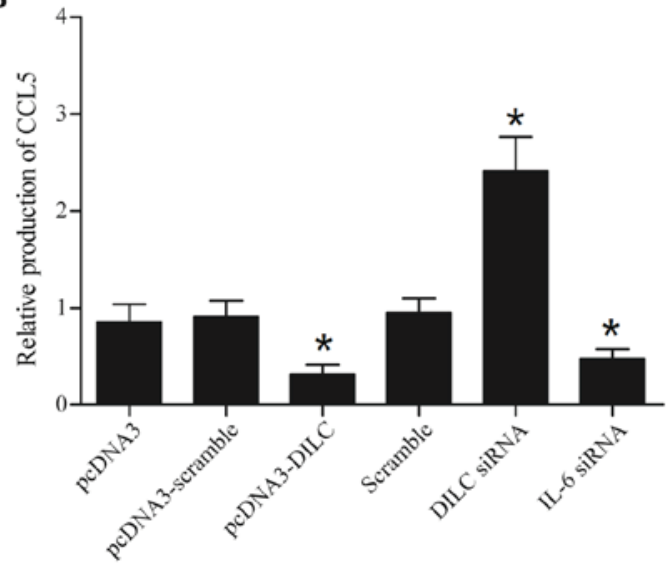

D

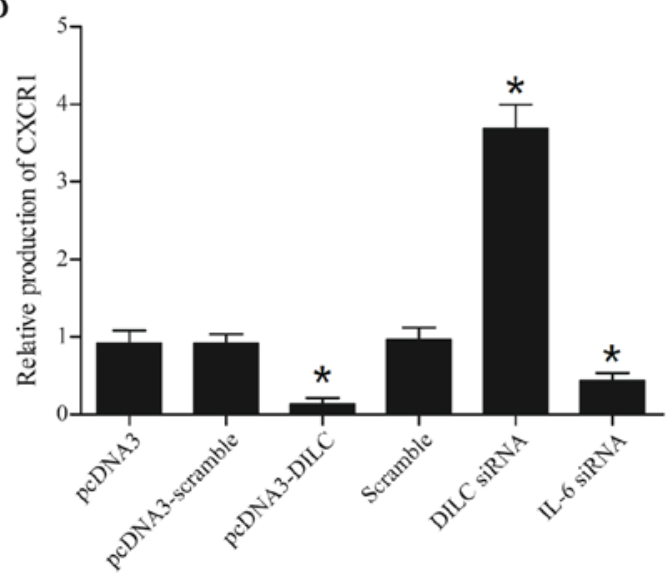

Figure 5. Alteration in TNF- $\alpha$, CCL5, E-selectin and CXCR1 expression in THP-1 cells treated with LPS, and transfected with plasmids containing DILC, DILC siRNA or IL-6 siRNA. (A) TNF- $\alpha$ mRNA expression levels in the DILC siRNA group were upregulated, and downregulated in the DILC and IL-6 siRNA treated groups. (B) DILC siRNA increased, and DILC and IL-6 siRNA decreased CCL5 expression levels. (C) E-selectin expression was enhanced in the DILC siRNA group, but inhibited in DILC and IL-6 siRNA groups. (D) CXCR1 mRNA expression levels in the DILC siRNA group were upregulated, but downregulated in DILC and IL-6 siRNA. "P<0.05 vs. pcDNA3. CCL5, chemokine ligand 5; CXCR1, C-X-C motif chemokine receptor 1; DILC, deleted in liver cancer 1; IL, interleukin; siRNA, short interfering RNA; TNF- $\alpha$, tumor necrosis factor- $\alpha$.

reduce the mRNA expression levels of vascular endothelial growth factor, IL-10 and IL-6 in human melanoma cells (27).
As a proinflammatory cytokine expressed by fibroblasts, endothelial cells and monocytes, IL-6 may activate T and 

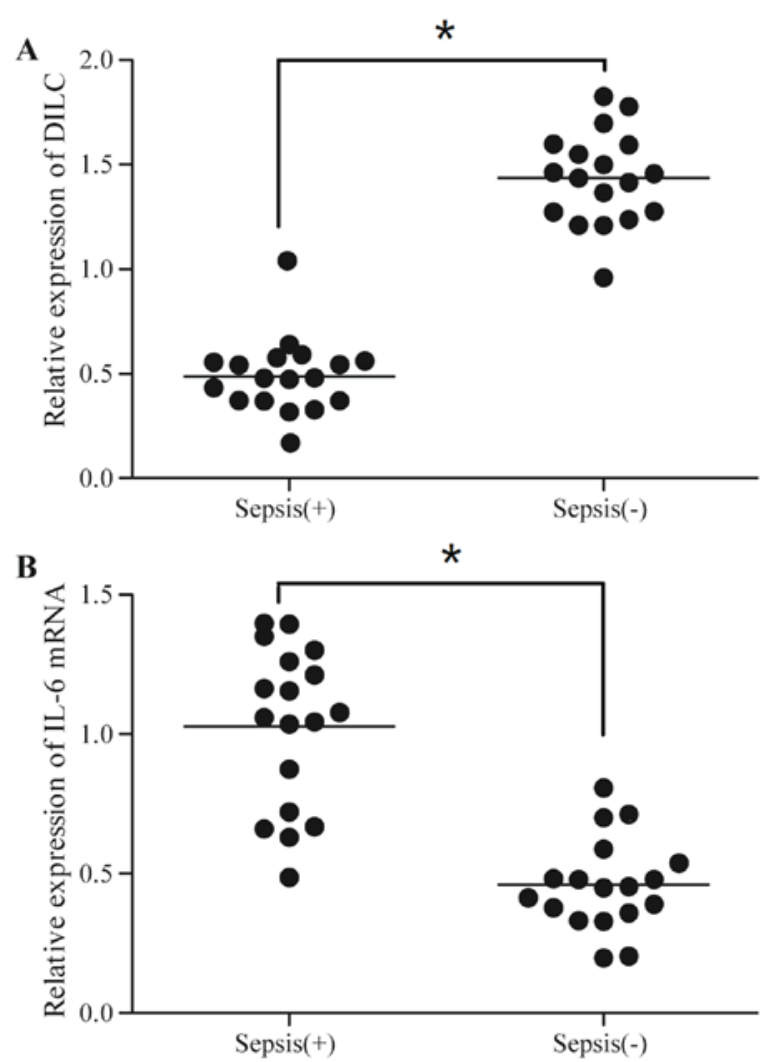

C

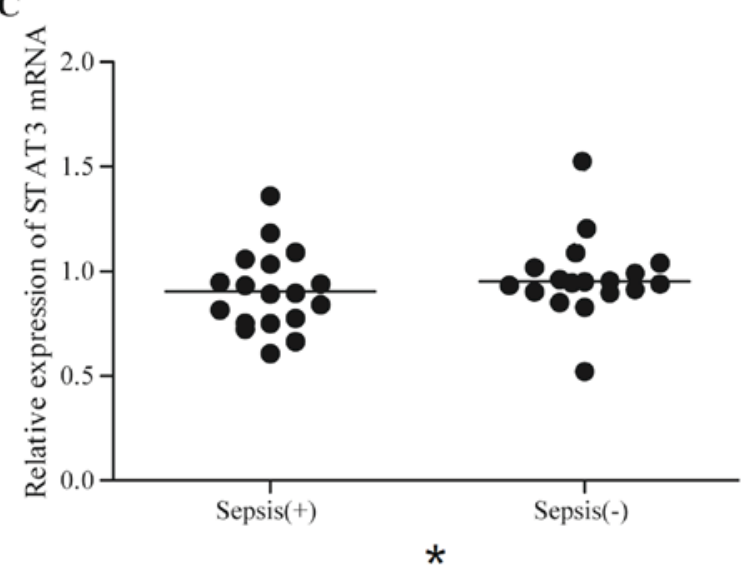

D

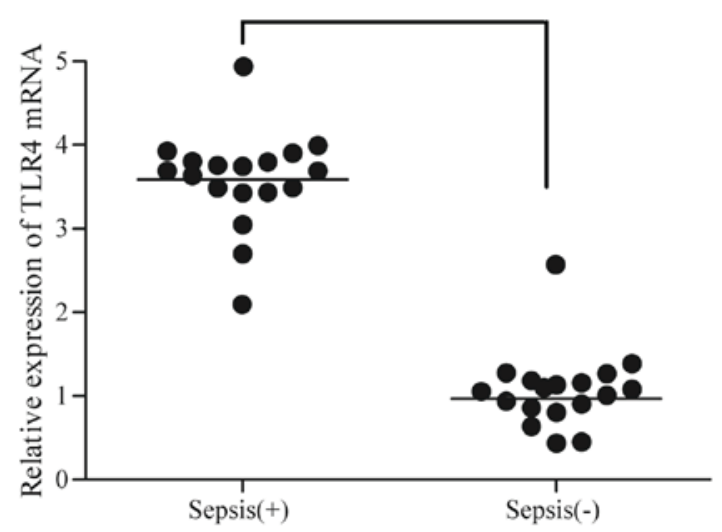

Figure 6. Expression of sepsis-associated genes including DILC, IL-6 mRNA, STAT3 and TLR4 vary between septic and healthy groups. (A) DILC was under-expressed in subjects with sepsis. (B) IL-6 mRNA was highly expressed in subjects with sepsis. (C) STAT3 expression levels in subjects with sepsis were not significantly different. (D) Subjects with sepsis exhibited higher expression levels of TLR4. "P<0.05. DILC, deleted in liver cancer 1; IL, interleukin; STAT3, signal transducer and activator of transcription 3; Toll-like receptor 4.
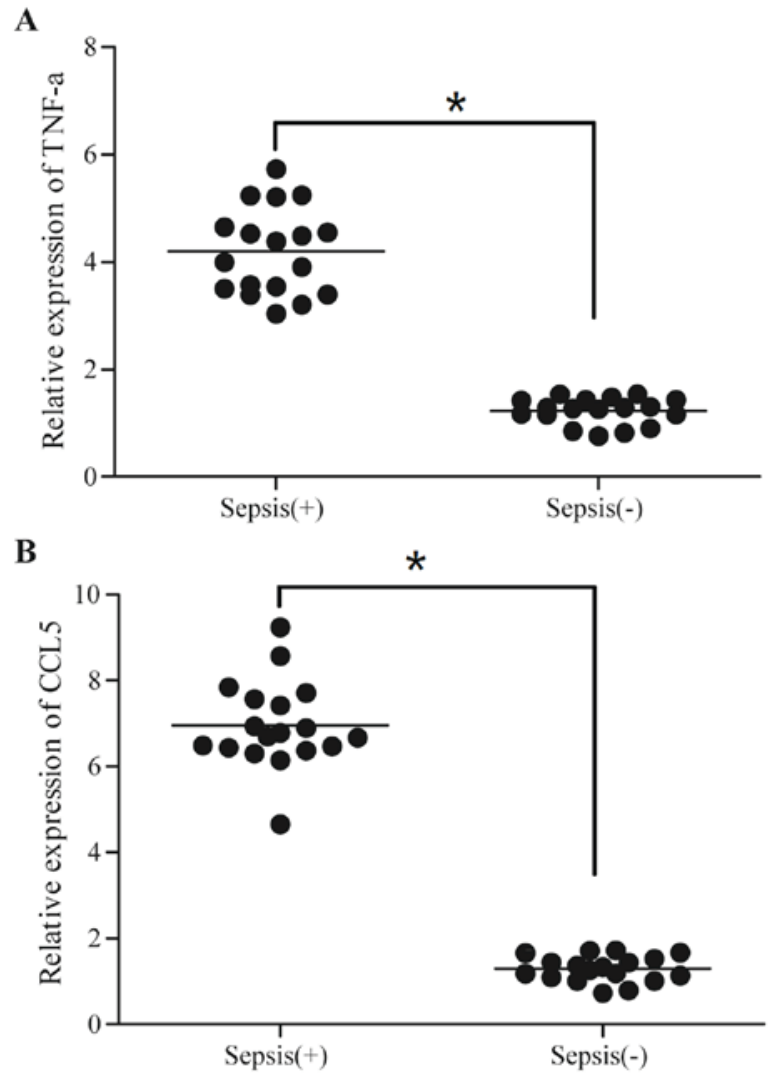

C

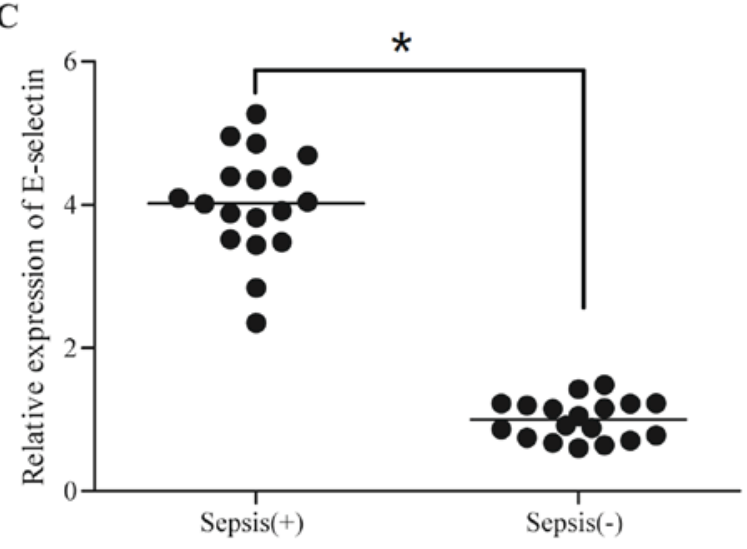

D

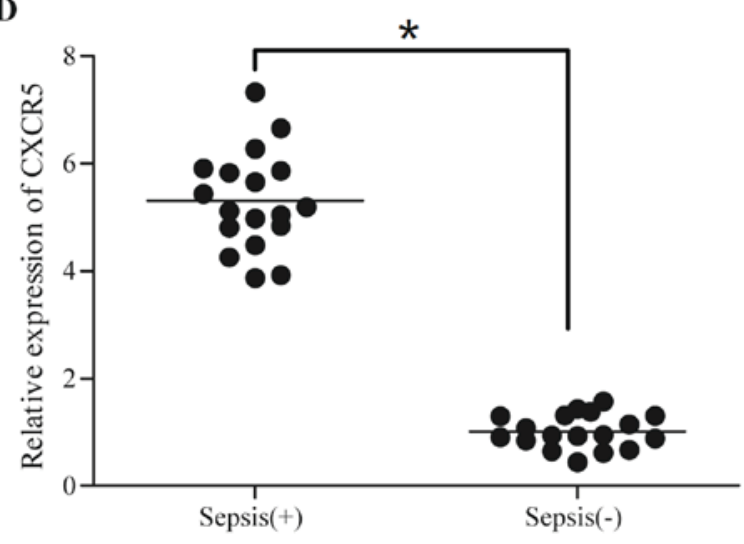

Figure 7. Expression of sepsis-associated genes including TNF- $\alpha$, CCL5, E-selection mRNA, and CXCR1 vary between septic and healthy groups. (A) TNF- $\alpha$ was highly expressed in subjects with sepsis. (B) CCL5 expression levels in subjects with sepsis were increased. (C) E-selectin mRNA was highly expressed in subjects with sepsis. (D) Subjects with sepsis exhibited a higher level of CXCR1. "P<0.05. CL5, chemokine ligand 5; CXCR1, C-X-C motif chemokine receptor 1 ; TNF- $\alpha$, tumor necrosis factor- $\alpha$. 
B lymphocytes, and induce fever (28). A previous report demonstrated that IL-6 may serve a critical role in inflammatory responses against the invasion of microbes (29). For example, a previous study indicated that a high concentration of IL-6 was associated with higher mortality and risk of severe sepsis (30), whereas the critical role of the IL-6 signaling pathway in systemic inflammation has been observed within IL-6-deficient mice (31).

As pattern recognition receptors, TLRs serve important roles in the mediation of innate immunity so as to provide protection against endotoxins (32). It has been demonstrated that LPS-induced TLR4 activation may trigger the phosphorylation of STAT3 primarily via myeloid differentiation primary response gene MyD88 (MyD88), an adaptor protein of TLR4. In addition, the suppression of $\mathrm{p} 38$, a downstream target of the TLR4 signaling, may lead to a decreased level of p-STAT3 at the tyrosine site, while p38 suppression and TLR4 deficiency may inhibit stress-induced DNA-binding activity and the phosphorylation of STAT3. Collectively, these data suggested a connection between STAT3 and TLR4/p38 in chronic stress (33), and it appears that TLR4/p38/IL-10 activates STAT3 following chronic stress (33). Previous studies have revealed that the expression levels of TLR4 are increased in the monocytes of patients with sepsis and healthy subjects challenged with LPS $(34,35)$. In addition, the stimulation of TLR4 may lead to the expression of anti- and proinflammatory factors by activating two distinct signaling pathways (36). In particular, TLR4 is a unique member of the TLRs due to its ability to mediate the Toll-interleukin receptor (TIR)-do main-containing-adapter-inducing interferon- $\beta$ (TRIF)- and MyD88-dependent pathways (37). Through TNF receptor-associated factor- 6 , interferon (IFN) $\beta$ and the TRIF-dependent signaling pathway are involved in the mediation of NF- $\mathrm{KB}$ activation (38). In addition, IFN- $\beta$ has been reported to be involved in the regulation of late-stage hyper-inflammation in sepsis (39).

LPS is secreted by gram-negative bacteria and is a pathogen-associated molecular pattern. Once recognized by TLR4, LPS is able to activate a signaling cascade and lead to the upregulated expression of certain cytokines that are associated with the onset and progression of sepsis (40). The majority of sepsis cases $(>50 \%)$ are triggered by gram-negative bacteria that are primarily recognized by TLR4 (1). For example, Tsujimoto et al (41) demonstrated that the serum levels of TLR4 protein increased markedly in patients with sepsis upon pathogen infection (41), and it has been suggested that the protein expression levels of TIR domain-containing adaptor protein and TLR4, rather than gene polymorphisms, may be associated with the severity of sepsis (42). LPS increased STAT3 mRNA expression, whereas DILC or IL-6 did not, suggesting that LPS did not increase STAT3 mRNA expression via the DILC/IL-6 signaling pathway (43).

In conclusion, the association between IncRNA DILC and sepsis was investigated; to the best of our knowledge, the present study was the first to demonstrate that DILC directly inhibited the expression of IL-6, which subsequently modulated TLR4-dependent inflammatory responses via STAT3. In addition, TLR4 may act as an important mediator of inflammatory responses in sepsis. Therefore, it was hypothesized in the present study that lnc-DILC may act as a potential prognostic marker of sepsis, and may additionally be considered to be a potential therapeutic target for the treatment of sepsis.

\section{Acknowledgements}

Not applicable.

\section{Funding}

The present study was supported by the Natural Science Foundation of Guangdong Province (grant no. S2013010016574), Pilot Projects of Clinical Medical Research and Transformation Center of Guanzhou City (grant no. 201508020005), and the National Key Clinical Specialty Construction Foundation (grant no. 2012-649).

\section{Availability of data and materials}

The datasets used and/or analyzed during the present study are available from the corresponding author on reasonable request.

\section{Authors' contributions}

WH planned the study, collected and analyzed the data, and prepared the manuscript; LH planned the study, collected and analyzed the data; MW collected, analyzed and interpreted the data, and prepared the manuscript; MF collected the literature and visualized the data; YD collected the data, and prepared the manuscript; and, HZ planned the study, organized the funding and prepared the manuscript. All authors read and approved the final manuscript.

\section{Ethics approval and consent to participate}

All data processing and sample collection were approved by the Ethics Committee of Guangdong Academy of Medical Sciences. The present study was performed according to the Declaration of Helsinki.

\section{Patient consent for publication}

Written informed consent was obtained from all patients.

\section{Competing interests}

The authors declare that they have no competing interests.

\section{References}

1. Angus DC, Linde-Zwirble WT, Lidicker J, Clermont G, Carcillo J and Pinsky MR: Epidemiology of severe sepsis in the United States: Analysis of incidence, outcome, and associated costs of care. Crit Care Med 29: 1303-1310, 2001.

2. Bateman BT, Schmidt U, Berman MF and Bittner EA: Temporal trends in the epidemiology of severe postoperative sepsis after elective surgery: A large, nationwide sample. Anesthesiology 112: 917-925, 2010.

3. Hamano K, Gohra H, Noda H, Katoh T, Fujimura Y, Zempo N and Esato K: Increased serum interleukin-8: Correlation with poor prognosis in patients with postoperative multiple organ failure. World J Surg 22: 1077-1081, 1998. 
4. Rubulotta FM, Ramsay G, Parker MM, Dellinger RP, Levy MM and Poeze M; Surviving Sepsis Campaign Steering Committee; European Society of Intensive Care Medicine; Society of Critical Care Medicine: An international survey: Public awareness and perception of sepsis. Crit Care Med 37: 167-170, 2009.

5. Kawamoto T, Ii M, Kitazaki T, Iizawa Y and Kimura H: Tak-242 selectively suppresses toll-like receptor 4-signaling mediated by the intracellular domain. Eur J Pharmacol 584: 40-48, 2008.

6. Biffl WL, Moore EE, Moore FA and Peterson VM: Interleukin-6 in the injured patient. Marker of injury or mediator of inflammation? Ann Surg 224: 647-664, 1996.

7. Pape HC, Schmidt RE, Rice J, van Griensven M, das Gupta R, Krettek $\mathrm{C}$ and Tscherne $\mathrm{H}$ : Biochemical changes after trauma and skeletal surgery of the lower extremity: Quantification of the operative burden. Crit Care Med 28: 3441-3418, 2000.

8. Giannoudis PV, Smith RM, Banks RE, Windsor AC, Dickson RA and Guillou PJ: Stimulation of inflammatory markers after blunt trauma. Br J Surg 85: 986-990, 1998.

9. Stensballe J, Christiansen M, Tønnesen E, Espersen K, Lippert FK and Rasmussen LS: The early IL-6 and IL-10 response in trauma is correlated with injury severity and mortality. Acta Anaesthesiol Scand 53: 515-521, 2009.

10. Adib-Conquy M and Cavaillon JM: Compensatory anti-inflammatory response syndrome. Thromb Haemost 101: 36-47, 2009.

11. Sander M, Irwin M, Sinha P, Naumann E, Kox WJ and Spies CD: Suppression of interleukin-6 to interleukin-10 ratio in chronic alcoholics: association with postoperative infections. Intensive Care Med 28: 285-292, 2002

12. Mercer TR, Dinger ME and Mattick JS: Long non-coding RNAs: Insights into functions. Nat Rev Genet 10: 155-159, 2009

13. Geisler S and Coller J: RNA in unexpected places: Long non-coding RNA functions in diverse cellular contexts. Nat Rev Mol Cell Biol 14: 699-712, 2013.

14. Ben-Neriah Y and Karin M: Inflammation meets cancer, with NF-kB as the matchmaker. Nat Immunol 12: 715-723, 2011.

15. Kagoya Y, Yoshimi A, Kataoka K, Nakagawa M, Kumano K, Arai S, Kobayashi H, Saito T, Iwakura Y and Kurokawa M: Positive feedback between NF-kB and TNF- $\alpha$ promotes leukemia-initiating cell capacity. J Clin Invest 124: 528-542, 2014

16. Wang X, Sun W, Shen W, Xia M, Chen C, Xiang D, Ning B, Cui X, Li H, Li X, et al: Long non-coding RNA DILC regulates liver cancer stem cells via IL-6/STAT3 axis. J Hepatol 64: 1283-1294, 2016

17. Greenhill CJ, Rose-John S, Lissilaa R, Ferlin W, Ernst M, Hertzog PJ, Mansell A and Jenkins BJ: IL-6 trans-signaling modulates TLR4-dependent inflammatory responses via STAT3. J Immunol 186: 1199-1208, 2011.

18. Livak KJ and Schmittgen TD: Analysis of relative gene expression data using real-time quantitative PCR and the 2(-Delta Delta C(T)) method. Methods 25: 402-408, 2001.

19. Li RJ, Gao CY, Guo C, Zhou MM, Luo J and Kong LY: The Anti-inflammatory Activities of two major withanolides from physalisminima via acting on NF- $\mathrm{B}$, STAT3, and HO-1 in LPS-stimulated RAW264.7 Cells. Inflammation 40: 401-413, 2017.

20. Schroeder A, Herrmann A, Cherryholmes G, Kowolik C, Buettner R, Pal S, Yu H, Müller-Newen G and Jove R: Loss of androgen receptor expression promotes a stem-like cell phenotype in prostate cancer through STAT3 signaling. Cancer Res 74: $1227-1237,2014$

21. Korkaya H, Kim GI, Davis A, Malik F, Henry NL, Ithimakin S, Quraishi AA, Tawakkol N, D'Angelo R, Paulson AK, et al: Activation of an IL6 inflammatory loop mediates trastuzumab resistance in HER2+ breast cancer by expanding the cancer stem cell population. Mol Cell 47: 570-584, 2012.

22. Jinushi M, Chiba S, Yoshiyama H, Masutomi K, Kinoshita I, Dosaka-Akita H, Yagita H, Takaoka A and Tahara H: Tumor-associated macrophages regulate tumorigenicity and anticancer drug responses of cancer stem/initiating cells. Proc Natl Acad Sci USA 108: 12425-12430, 2011.

23. He G, Dhar D, Nakagawa H, Font-Burgada J, Ogata H, Jiang Y, Shalapour S, Seki E, Yost SE, Jepsen K, et al: Identification of liver cancer progenitors whose malignant progression depends on autocrine IL-6 signaling. Cell 155: 384-396, 2013.
24. Huang WL, Yeh HH, Lin CC, Lai WW, Chang JY, Chang WT and Su WC: Signal transducer and activator of transcription 3 activation up-regulates interleukin-6 autocrine production: A biochemical and genetic study of established cancer cell lines and clinical isolated human cancer cells. Mol Cancer 9: 309, 2010.

25. Judd LM, Bredin K, Kalantzis A, Jenkins BJ, Ernst M and Giraud AS: STAT3 activation regulates growth, inflammation, and vascularization in a mouse model of gastric tumorigenesis Gastroenterology 131: 1073-1085, 2006.

26. Ogura H, Murakami M, Okuyama Y, Tsuruoka M, Kitabayashi C, Kanamoto M, Nishihara M, Iwakura Y and Hirano T: Interleukin-17 promotes autoimmunity by triggering a positive-feedback loop via interleukin-6 induction. Immunity 29 : 628-636, 2008

27. Sumimoto $\mathrm{H}$, Imabayashi $\mathrm{F}$, Iwata $\mathrm{T}$ and Kawakami $\mathrm{Y}$ : The BRAF-MAPK signaling pathway is essential for cancer-immune evasion in human melanoma cells. J Exp Med 203: 1651-1656, 2006.

28. Gao JW, Zhang AQ, Pan W, Yue CL, Zeng L, Gu W and Jiang J: Association between IL-6-174G/C polymorphism and the risk of sepsis and mortality: A systematic review and meta-analysis. PLoS One 10: e0118843, 2015.

29. Borden EC and Chin P: Interleukin-6: A cytokine with potential diagnostic and therapeutic roles. J Lab Clin Med 123: 824-829, 1994.

30. Uusitalo-Seppälä R, Koskinen P, Leino A, Peuravuori H, Vahlberg T and Rintala EM: Early detection of severe sepsis in the emergency room: Diagnostic value of plasma C-reactive protein, procalcitonin, and interleukin-6. Scand J Infect Dis 43: 883-890, 2011

31. Jin YH, Hou W, Kang HS, Koh CS and Kim BS: The role of interleukin- 6 in the expression of PD-1 and PDL-1 on central nervous system cells following infection with Theiler's murine encephalomyelitis virus. J Virol 87: 11538-11551, 2013.

32. Takeda $\mathrm{K}$ and Akira S: Toll-like receptors in innate immunity. Int Immunol 17: 1-14, 2005.

33. Hu D, Wan L, Chen M, Caudle Y, LeSage G, Li Q and Yin D: Essential role of IL-10/STAT3 in chronic stress-induced immune suppression. Brain Behav Immun 36: 118-127, 2014.

34. Wittebole X, Coyle SM, Kumar A, Goshima M, Lowry SF and Calvano SE: Expression of tumour necrosis factor receptor and Toll-like receptor 2 and 4 on peripheral blood leucocytes of human volunteers after endotoxin challenge: A comparison of flow cytometric light scatter and immunofluorescence gating. Clin Exp Immunol 141: 99-106, 2005.

35. Härter L, Mica L, Stocker R, Trentz O and Keel M: Increased expression of toll-like receptor-2 and -4 on leukocytes from patients with sepsis. Shock 22: 403-409, 2004.

36. Hoebe K, Janssen E and Beutler B: The interface between innate and adaptive immunity. Nat Immunol 5: 971-974, 2004.

37. Kawai T and Akira S: The role of pattern-recognition receptors in innate immunity: Update on Toll-like receptors. Nat Immunol 11: 373-384, 2010.

38. Kumar V and Sharma A: Innate immunity in sepsis pathogenesis and its modulation: New immunomodulatory targets revealed. J Chemother 20: 672-683, 2008.

39. Weighardt H, Kaiser-Moore S, Schlautkötter S, Rossmann-Bloeck T, Schleicher U, Bogdan C and Holzmann B: Type I IFN modulates host defense and late hyperinflammation in septic peritonitis. J Immunol 177: 5623-5630, 2006.

40. Namath A and Patterson AJ: Genetic polymorphisms in sepsis. Crit Care Clin 25: 835-856, x, 2009.

41. Tsujimoto H, Ono S, Efron PA, Scumpia PO, Moldawer LL and Mochizuki H: Role of Toll-like receptors in the development of sepsis. Shock 29: 315-321, 2008.

42. Zhang J, Yang J, Xu X, Liang L, Sun H, Liu G, Zhang L and Su Y: The influence of genetic polymorphisms in TLR4 and TIRAP, and their expression levels in peripheral blood, on susceptibility to sepsis. Exp Ther Med 11: 131-139, 2016.

43. Liu X, Su X, Xu S, Wang H, Han D, Li J, Huang M and Cao X: MicroRNA in vivo precipitation identifies miR-151-3p as a computational unpredictable miRNA to target Stat 3 and inhibits innate IL-6 production. Cell Mol Immunol 15: 99-110, 2018. 